\title{
Effect of Bi-axial Residual Stresses on the Micro-Indentation Behaviour of Bulk Materials
}

\author{
Prakash Jadhav \\ Department of Mechanical Engineering \\ CMR Institute of Technology, Bangalore, India \\ Prakash.k@cmrit.ac.in
}

\begin{abstract}
Residual stresses can be of great importance in bulk materials and coatings with respect to their effect on the mechanical integrity and reliability. High residual stresses cause degradation of mechanical properties and challenge the reliability (fatigue, fracture, corrosion and wear) of the bulk materials and coatings. Compared to traditional techniques, the depth-sensing indentation technique provides a quick and effective method to measure in-plane residual stresses. Literature shows that contact hardness, stiffness and indentation work (obtained from indentation load-displacement curves) are sensitive to the residual stress. A new experimental technique to verify the numerical simulations is presented here. It involves conducting measurements of residual stresses using depth sensing micro-indentation and studying its effect on the load-displacement behaviour. A special bi-axial loading fixture is designed and developed to apply controlled biaxial tensile/compressive stresses on the specimen simulating in-plane residual stresses. In-plane residual stresses are measured using strain measurement system. Instrumented micro-indentation system is used to obtain the load-displacement behaviour for variable biaxial loading on the specimen. It has been shown here that residual stress field affects the indentation behaviour of the bulk materials.
\end{abstract}

Keyword: Residual stress, Indentation

\section{INTRODUCTION}

It is a well known fact that the residual stress affects the mechanical integrity and reliability of bulk materials and coatings ultimately causing fatigue, fracture, corrosion and wear type of failure. Thermal barrier coatings generates residual stresses (commonly introduced by thermal mismatch or mechanical and thermal processing) which affects the performance of mechanical structures. For a reliable engineering design, the actual level and sign of residual stress in the specimen must be determined, frequently by measurements, since associated material properties are many times unknown. Depth sensing indentation technique is a novel technique developed in past two decades for measuring mechanical properties of materials such as Young's modulus E and yield strength $\sigma_{\mathrm{y}}$ and hardness for most engineering materials [1-2]. Different factors influencing the measurement of mechanical properties by indentation are evaluated by few researchers like Bolshakov, Pharr, Hey and Beck [3-6]. Some of them have tried to use this technique to measure residual stresses in bulk materials experimentally and analytically. Most notables of them who used experimental technique are Swadner, Tsui, Carlsson, Lee [7-12] and the one who used analytical technique are Bolshakov, Carlsson and Chen [13-16].

This experimental investigation is based on the earlier simulation attempt by Chen [16] to extend the usefulness of this method to measure the residual stress, $\sigma_{\text {res. }}$ In this study, the finite element analysis was used to investigate the effect of in-plane equi-biaxial residual stress on hardness and stiffness measured from indentation tests on a bulk material (or thick coating). By varying the material parameter and residual stress over a large range, it was found that both hardness and stiffness vary with residual stress, especially for high-strength materials with large yield strain [Fig. 1]. Both, the plastic pile-up (or elastic sink-in) and indentation work were found to be very sensitive to the level of residual stress, which makes the determination of residual stress from indentation test possible. Based on the functional forms of normalized contact hardness, stiffness, and indentation work, a reverse algorithm was presented which may be used to measure the material yield stress, 
modulus, and residual stress from one simple indentation test. The comparison between the material properties predicted from reverse analysis and the input parameters used in numerical indentation experiments showed good agreement. The effects of residual stress on indentation stress and plastic zone was also investigated. It was found that the indentation compressive stress diminishes with residual tension, which leads to an enlarged plastic zone and gives apparent lower hardness. On the other hand, with residual compression the maximum indentation compressive stress was increased, causing less plastic deformation (with smaller plastic zone) and gave rise to the hardness. It was also found that the elastic recovery upon unloading is very large for materials with high yield strain, and the elastic recovery increases with increasing residual compression. Unlike many of the previous studies, the new indentation technique proposed in this paper did not require a reference stress-free material for comparison purposes. The present study attempts to validate the results of the simulation study.

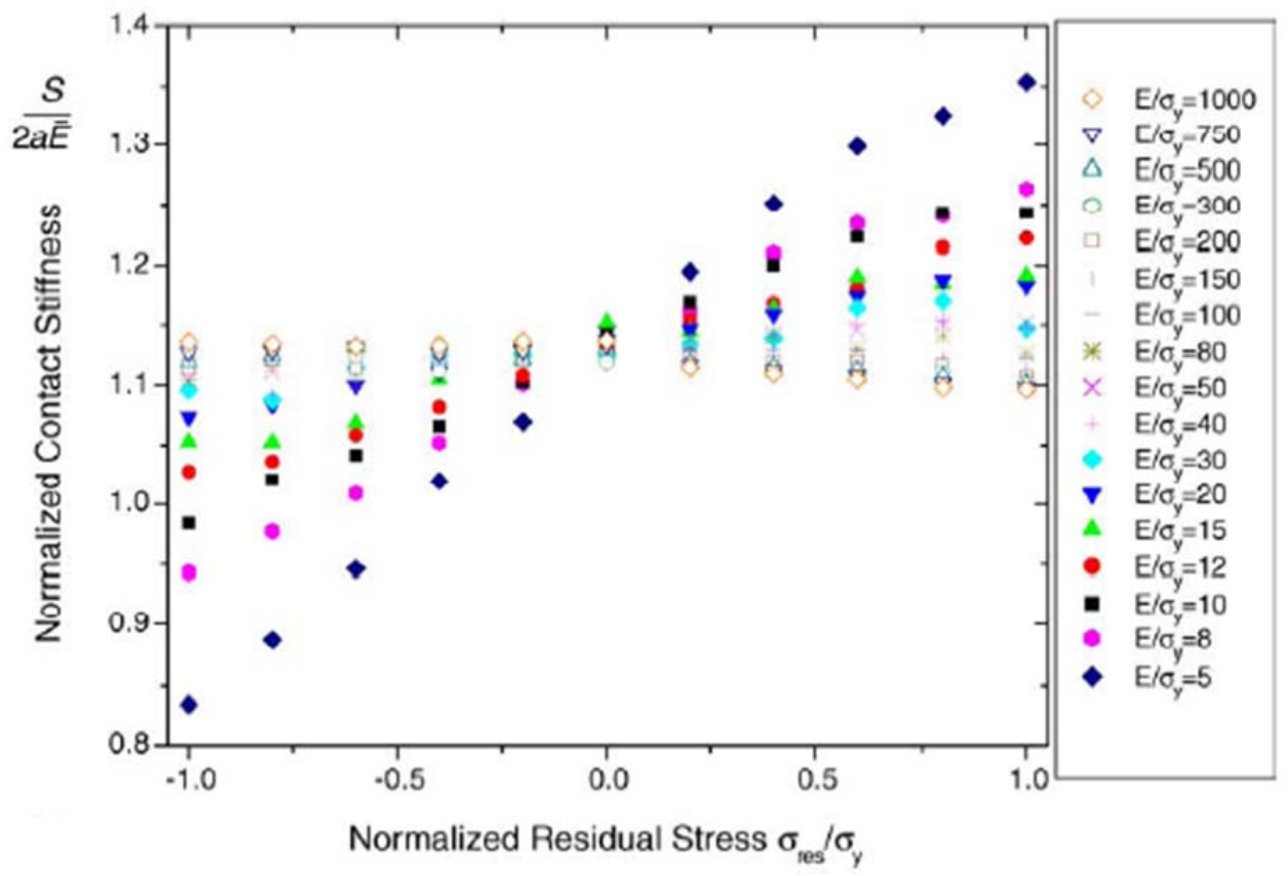

Fig 1: Residual stress effects on contact stiffness [Ref 16]

\section{REVIEW OF INDENTATION TECHNIQUE}

The indentation technique is based on high resolution instruments that continuously monitor the load and displacements of indenter as it is pushed into and withdrawn from the material. The load-displacement curve obtained contains valuable information like elastic/plastic deformation which enables to derive mechanical properties from it. Instrumented indentation is characterized by a sharp rigid indenter (with a half apex angle $\alpha$ ) penetrating normally into a homogeneous solid where the indentation load, $P$, and displacement, $\delta$, are continuously recorded during one complete cycle of loading and unloading (Fig. 2a,b). In this case, the conical indenter used has a rigid cone with half cone angle $\alpha=60^{\circ}$. By neglecting friction and the finite compliance of the measuring system and the indenter tip, the equations used to extract the hardness $H$ and indentation modulus $M$ are

$$
H=P / A=c_{b} \sigma_{y}
$$

and

$$
S=\gamma \beta \frac{2}{\sqrt{\pi}} M \sqrt{A}
$$



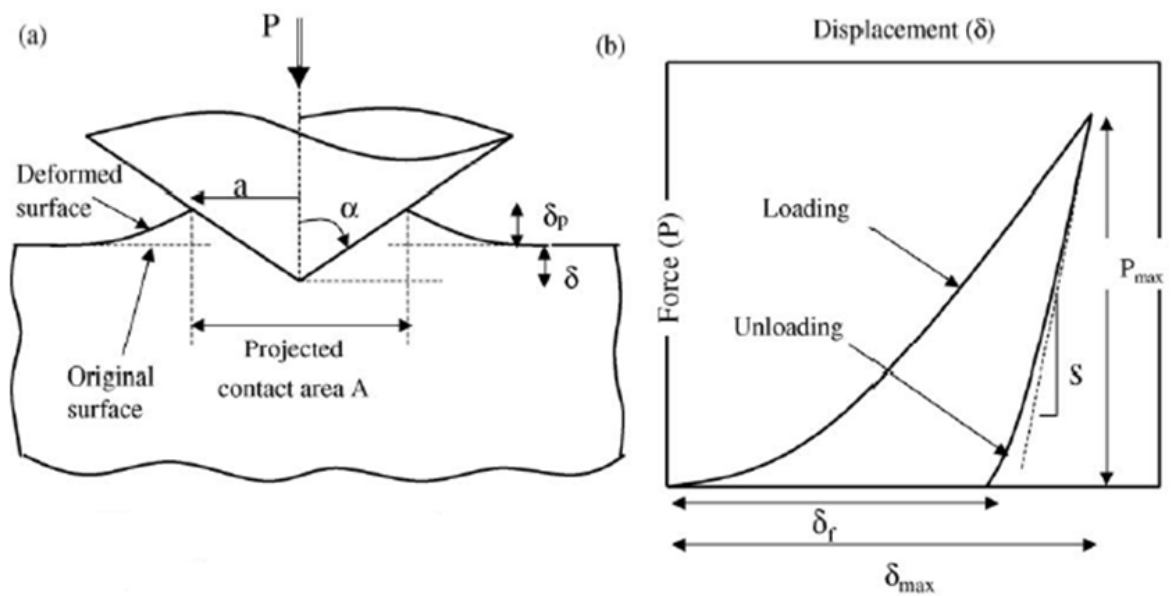

Fig 2: Indentation - a. contact area and contact depth notations, b. load-displacement plot [Ref 16]

Here, the hardness $H$ is defined as the ratio between indentation load $P$, projected contact area $A$ and yield stress $\sigma_{y}$. The indentation modulus $M$ is given by the plane-strain modulus, $\bar{E} \equiv E /\left(1-v^{2}\right)$, for isotropic materials and by a more complicated weighted average of the elastic constants for anisotropic materials, where $E$ and $v$ are the Young's modulus and Poisson's ratio of the bulk material, respectively. The contact stiffness $S=d P / d \delta$ is obtained from the slope of initial portion of the elastic unloading curve (Fig.2b).

As the indenter penetrates the specimen, the materials either produce plastic pile-up at the crater rim (when the yield strain, $\sigma_{y} / E$, is small), or exhibit the elastic sink-in effect (when $\sigma_{y} / E$ is large). The amount of pileup/sink-in is denoted as $\delta_{p}$. For conical indenters, the projected contact area $A$ is given by:

$$
A=\pi a^{2}=\pi(\tan \alpha)^{2} \delta_{c}^{2}=24.5 \delta_{c}^{2},
$$

where the contact depth,

$$
\delta_{c}=\delta+\delta_{p}
$$

Eq. (4) contains contributions of both plastic pile-up around the indenter and elastic sink-in, which is counted negative. It is obvious from Eqs. (3) and (4), that the projected contact area depends on $\delta_{p}$. Oliver and Pharr [1-2] proposed an elastic model for determining the contact area in which plastic pile-up is neglected:

$$
\left|\delta_{p}\right|=\varepsilon P_{\max } / S_{\max },
$$

where $\varepsilon=0.75$ for a conical indenter. Alternatively, the pile-up and contact area can be measured experimentally or determined from the numerical analysis (such as the finite element method or molecular dynamics). Once $A$ is determined properly, the hardness is then obtained from Eq. (1) and the stiffness can be derived from Eq. (2), which allows one to measure $\bar{E}$ and $\sigma_{y}$ by using the indentation technique.

\section{EXPERIMENTS}

Indentation experiments were conducted in a specially designed test set up as shown in the Figure 3. A MTS 858 mini-bionix instrumented test system was used to perform indentation testing and acquire the load displacement data. A specially designed biaxial loading fixture [17] was mounted on the base plate of machine. The fixture has two screw driven loading systems to apply in-plane tensile/compressive load to the specimen in $\mathrm{x}$ and $\mathrm{y}$ direction and indentation test was conducted in vertical $\mathrm{z}$ direction. Anticlockwise rotation of screw applies in-plane compressive loading and clockwise rotation of screw applies in-plane tensile loading to the specimen. To evaluate large range of modulus to yield strength ratio materials, common commercial Aluminium alloys like 1100-H14, 5052-H32, 6061-T6, 7075-T6 and common plastics like polycarbonate and nylon were 
used for the specimen materials [Fig. 4]. Two ends of the octagonal specimens are clamped to the fixture using set screws and remaining two ends are clamped to the moving members of the loading system. The whole upper plate having specimen, can slide on the bottom plate which is fixed to base of test system to allow specimen movement in one axis enabling test at different locations. A Rockwell testing indenter (Newage make model 8110C) with 120 degree cone angle and with a diamond tip was used for Micro-indentation tests. General purpose uni-axial strain gages (Vishay Inc, EA-13-125AD-120) were installed on the specimens to measure the in-plane residual stresses in $\mathrm{x}$ and $\mathrm{y}$ axis directions. The axial force, displacement and strain data was acquired using MTS Flex SE controller. The strain signal was processed through digital strain indicator using half bridge circuit (Vishay Inc, P3) [Fig. 5]. In addition to the load and displacement data generated by load cell and LVDT in MTS system, additional high resolution laser displacement sensor (Micro-Epsilon make opto-control 1200-2) was also used as shown in the set up. Laser band transmitted by transmitter head is absorbed by receiving head of receiver unit. Vertical movement of indenter is calibrated in terms of percentage blockage of laser band by edge of indenter. Octagonal specimens of $72 \times 72 \times 3 \mathrm{~mm}$ size approximately were used for indentation tests. Total 12 specimens were tested for each material (uni-axial tensile and compressive loading and bi-axial tensile and compressive loading). The specimen surfaces to be indented were mechanically polished with successively finer grit materials to create a smooth surface for micro-indentation test and for installing strain gages.

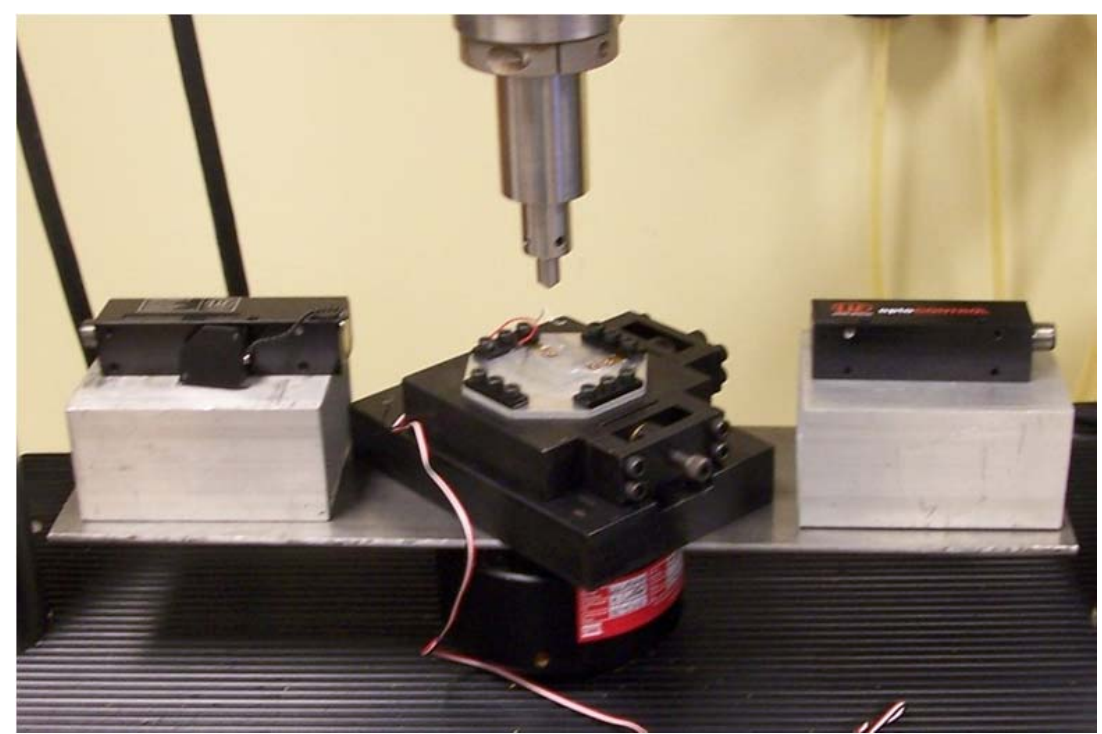

Fig 3: Indentation test system configuration

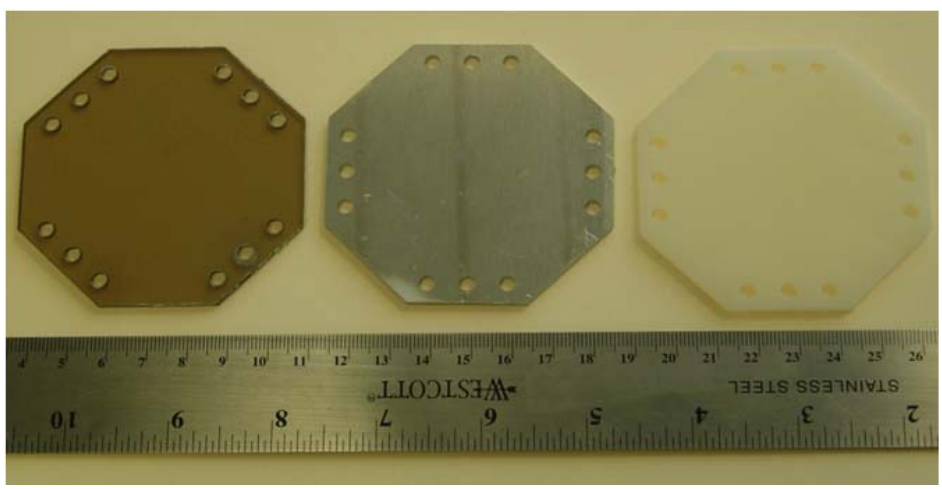

Fig 4: Specimens for indentation testing 


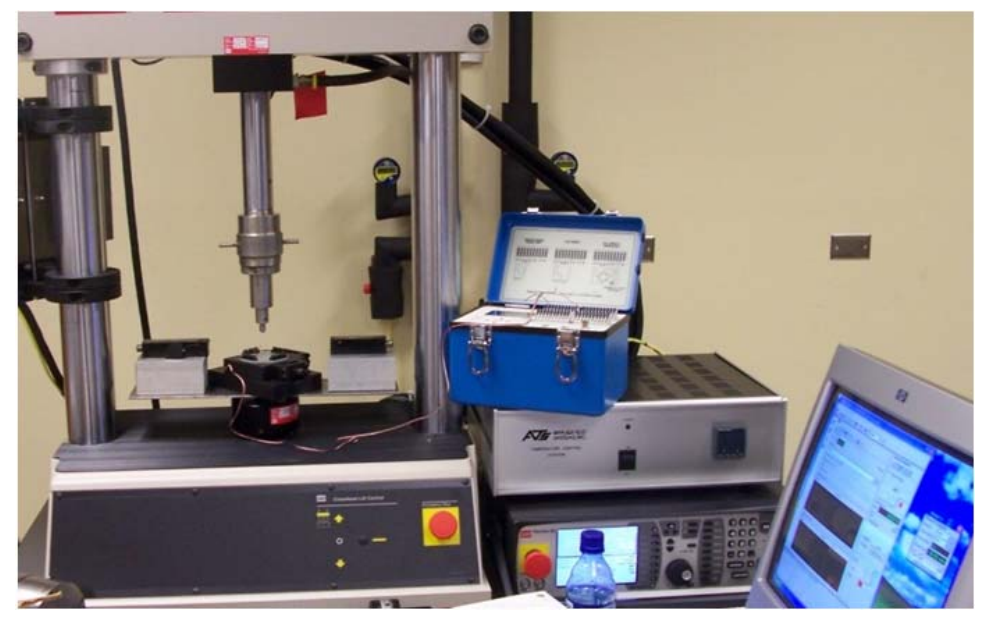

Fig 5: Indentation test set up with strain indicator

\section{DESIGN PARAMETERS}

The new experimental indentation technique used here to measure the mechanical properties has presented many problems. For designing the micro-indentation experiment which can measure in-plane residual stresses, various factors had to be considered like biaxial loading fixture, specimen, instrumented test system, strain measurement system and others. Experiment design considerations are discussed here.

The shape of the indenter is very important in indentation testing. The indenter can be spherical, pyramid or conical. Change in shape of hardness impression due to elastic recovery in unloading process causes non linearity in unloading curves. A geometric parameter $\varepsilon$, which varies from 0.74 to 0.79 , is used to estimate correct modulus and hardness. For the study presented here conical indenter with $60^{\circ}$ half included angle is used. For conical indenter pile-up of material around the contact impression causes errors in contact area estimation and in turn errors in modulus and hardness measurement by as much as $50 \%$ [4]. The pile-up errors can be removed by the use of load over stiffness squared parameter which is independent of contact area. As the specimens used here are only $3 \mathrm{~mm}$ thick, the specimen bending could cause errors in displacement measurement. These errors were removed by using thick rigid support for the thin specimens and the tightly clamping the specimen at the ends. As the proposed depth of indentation tests is less than $0.3 \mathrm{~mm}$, the size effect for thin specimens is negligible. Some of these errors can be removed by using high resolution sensor for displacement measurement.

The trial simulations were performed on the specimen model in FEA to study the stress contour pattern in the central specimen region due to applied tensile/compressive loading. Constant stress contours were found in the interest area of specimen where indentation test would be carried out and strain gauges will be installed. The specimen boundary condition effect has been studied and it was found that peak load (and in turn hardness) is sensitive to changes in specimen boundary condition, however, contact stiffness does not change with it.

The main cause of underestimation of modulus in micro-indentation testing is the machine compliance or load frame compliance $[1-2,5]$. In case of higher indentation depth tests, compliance of system set up comes into picture and causes errors in displacement measurements. The calibration for the load frame compliance can be performed using the following equation for total compliance $\mathrm{C}$ [1-2].

$$
C=C r+\frac{\sqrt{\pi}}{2 E e f f} \frac{1}{\sqrt{A}}
$$

If area function is known, the load frame compliance $\mathrm{C}_{\mathrm{f}}$ can be determined as the intercept of a plot of $\mathrm{C}$ vs $\mathrm{A}^{-}$ 1/2. At shallow depths contact compliance dominates total compliance while at larger depths the load frame compliance becomes the dominant factor. The accurate measurement of load frame compliance becomes essential for larger depths. One of the most important improvements in the calibration procedure was suggested by Oliver and Pharr. The ratio of load to the stiffness squared, $\mathrm{P} / \mathrm{S}^{2}$, is a directly measurable experimental parameter that is independent of the penetration depth or contact area. 


$$
\frac{P}{S^{2}}=\frac{\pi}{(2 \beta)^{2}} \frac{H}{E^{2}}
$$

Area independence of $\mathrm{P} / \mathrm{S}^{2}$, helps in determining machine compliance or load-frame compliance. At larger contact depths, $\mathrm{P} / \mathrm{S}^{2}$ should be constant. Another useful feature of $\mathrm{P} / \mathrm{S}^{2}$ is that it does not depend on the pile-up or sink-in behaviour. If modulus is known, above equation can be used to find hardness and vice versa irrespective of the amount of pile-up.

Use of rigid and stiffer components in the test system reduces the machine compliance effect in a considerable extent. Finally last but not the least the test equipment should meet the following criteria [5]. The tolerance for the force measurement should be less than $1.5 \%$. The error in the indentation depth measurement should be less than $1 \%$. The repeatability of testing equipment should be less than $5 \%$. The error of testing equipment should be smaller than $5 \%$. The uncertainty of test result depends on the accuracy of testing equipment, on the properties of testing item and on the testing range as well. Surface roughness has a strong influence too.

\section{RESULTS AND DISCUSSIONS}

\section{A. Experimental Errors}

Figure 6 shows the typical indentation behaviour (load-displacement plot) obtained from trial indentation tests of $5 \mathrm{~mm}$ thick aluminium 6061 plate at different indentation depths. The indentation modulus (measured from the slope of unloading curve) seems to decrease with increasing indentation depth. At lower indentation depth the modulus estimation seems to be closer to correct value. The test system compliance (load-frame compliance) seems to affect the indentation behaviour at higher indentation depths. A trial study showed that use of soft materials like aluminium as the load frame components introduces compliance in the system. Therefore highly stiff materials like steel were used in manufacturing the load frame components. An attempt has been made to estimate experimental load frame compliance by plotting the total compliance against the contact area as shown in equation 6 for the same aluminium specimen test results (Fig. 7). The y-intercept of the plot is the experimental load frame compliance. Corresponding load over stiffness squared parameter values were also plotted against the indentation depths in Fig. 8 where non-linearity of the plot is clearly visible. The correct value of the experimental load frame compliance would be the one which provides almost constant $\mathrm{P} / \mathrm{S}^{2}[1]$. The experimental load frame compliance was varied arbitrarily from its original value to get the constant load over stiffness parameter plot. As can be seen from the Figures 6 and 7, corrected load frame compliance $(25 \%$ of experimental value) provides constant load over stiffness squared value against indentation depth. This correct value of load frame compliance can be used for estimation of correct modulus at higher indentation depths using equations 6 and 7 .

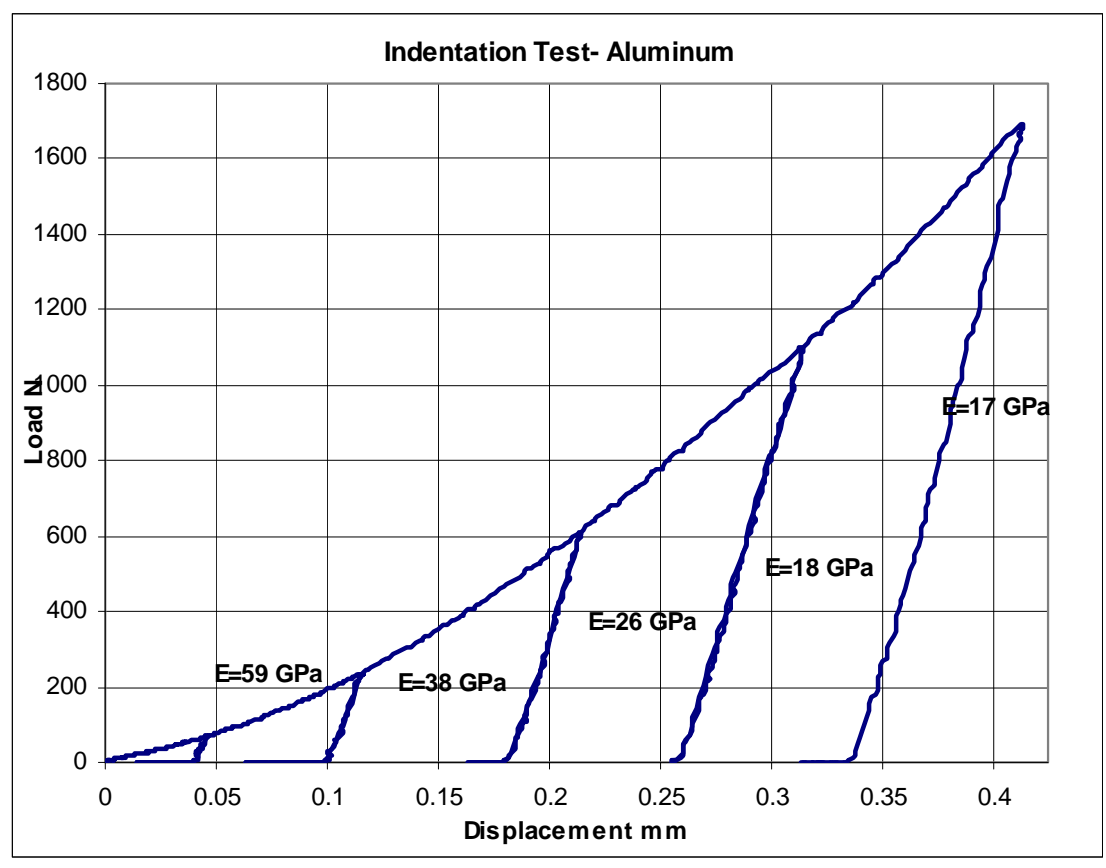

Fig 6: Compliance effect on indentation behaviour of Aluminium 6061 


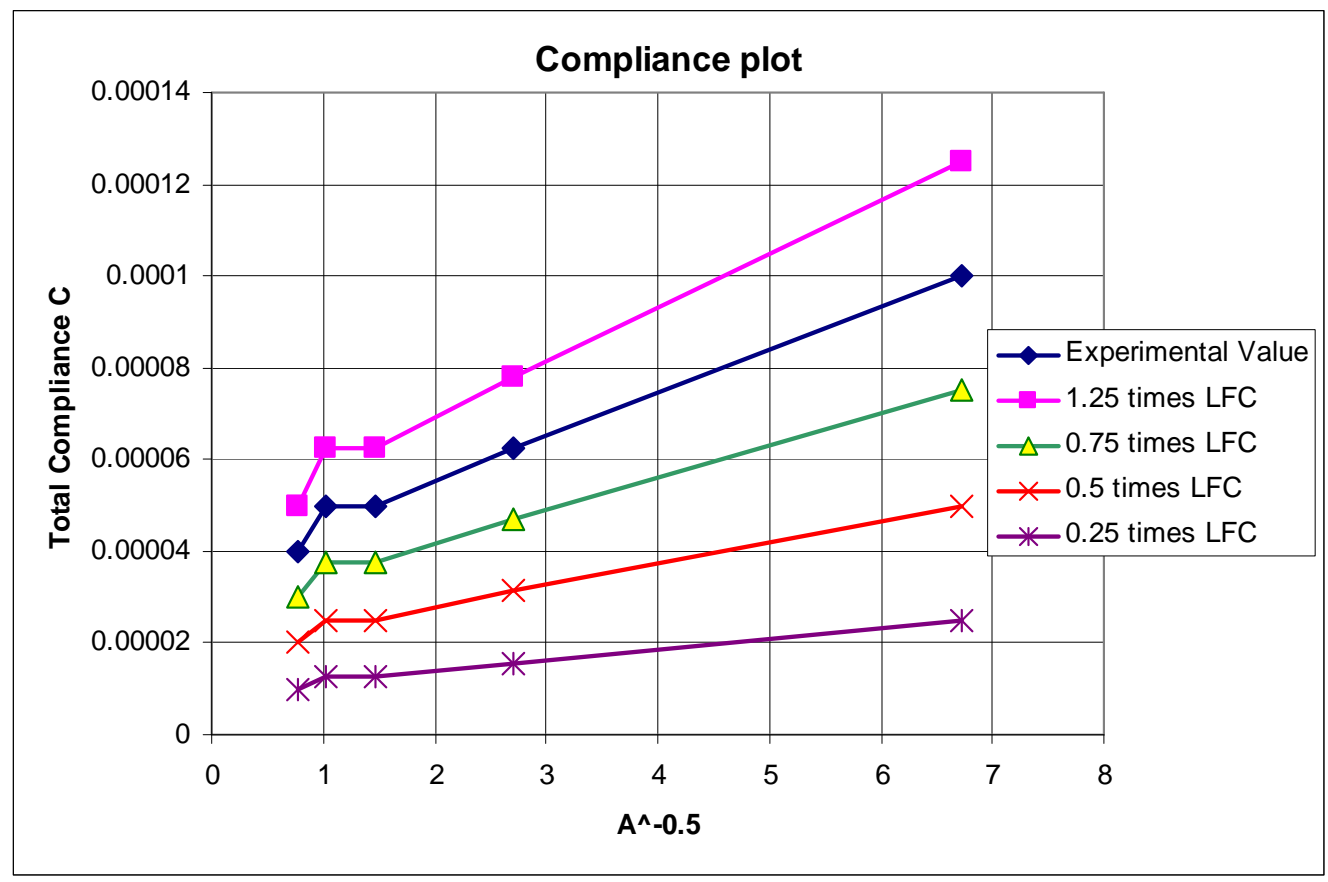

Fig 7: Compliance plot (Y-intercept is load frame compliance-LFC)

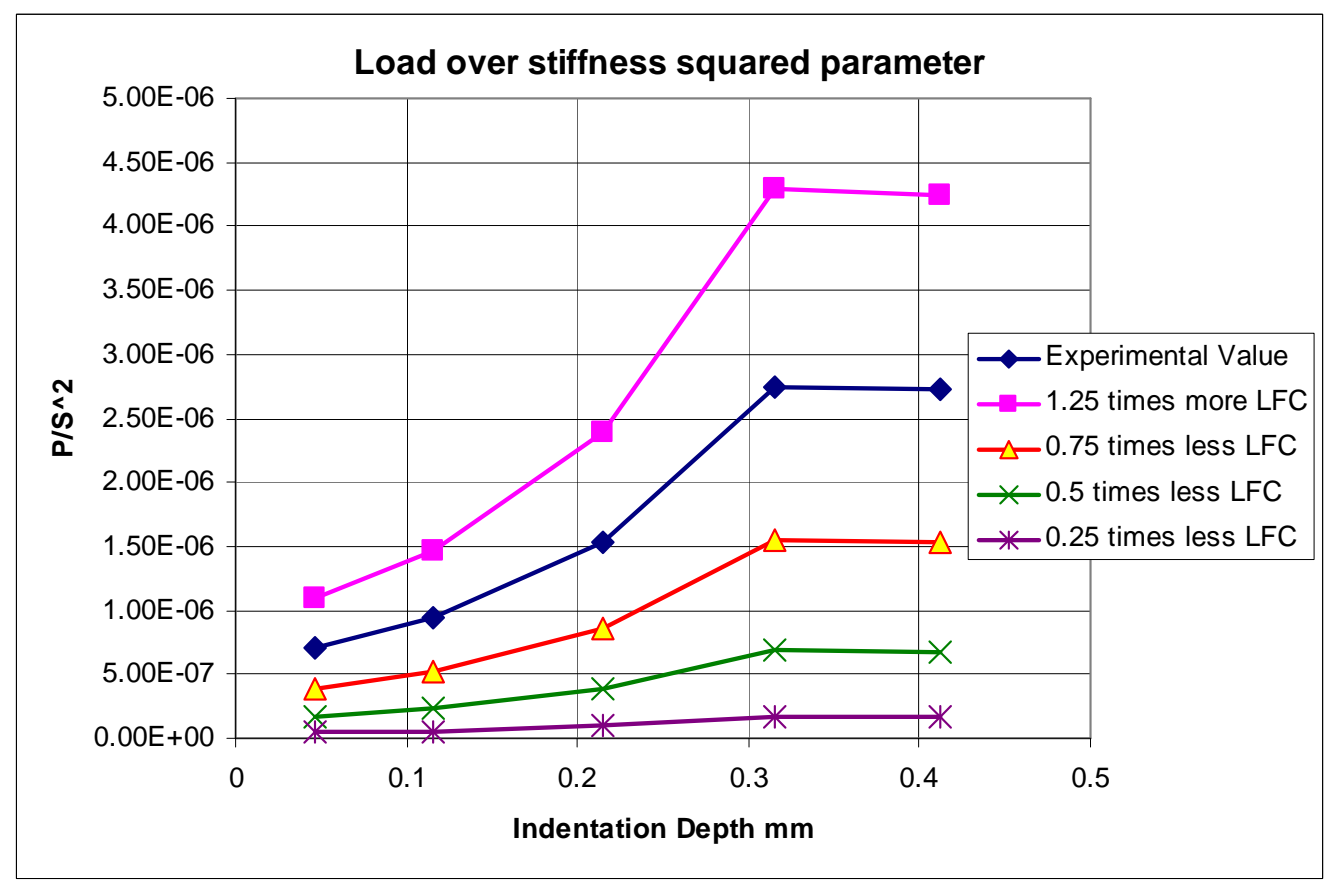

Fig 8: Load over stiffness squared parameter

\section{B. Residual Stress Effects}

As seen in Figure 9 and 10, tests were conducted on different materials like PC, Nylon and different grades of Aluminium (with increasing order of yield strength to get different modulus to yield strength ratios) to study the effect of induced residual stresses. In-plane biaxial tensile and compressive loading was applied using the fixture as shown earlier. These indentation tests were conducted for range of indentation depths $0.05,0.1$ and $0.2 \mathrm{~mm}$. Hardness and contact stiffness were computed from the experimental load displacement plots and normalized using equations mentioned by Chen [16]. The hardness was normalized by yield stress and contact stiffness was normalized by modulus of the material. The residual stress was also computed from the experimentally measured strain and normalized with yield stress. It was noted that indentation test depth has no 
real effect on the increasing or decreasing trend of normalized hardness and contact stiffness. Only effect it has is on the amplitude of these parameters. As can be seen from Figure 9 and 10, the contact stiffness and hardness do vary with the variation of the residual stress in the material. For the low yield strength materials, as the residual stress increases, hardness and contact stiffness increases. For the high yield strength material, as the residual stress increases, hardness and contact stiffness kind of levels off (does not change significantly). There are slight experimental errors which will be removed completely in the next set of experiments. Thus we can say that indentation behaviour is greatly affected by the internal residual stress in the material and hence it can be safely said that for any material, especially thermal barrier coatings, just a simple indentation test response can tell the existence of level of residual stresses in it.

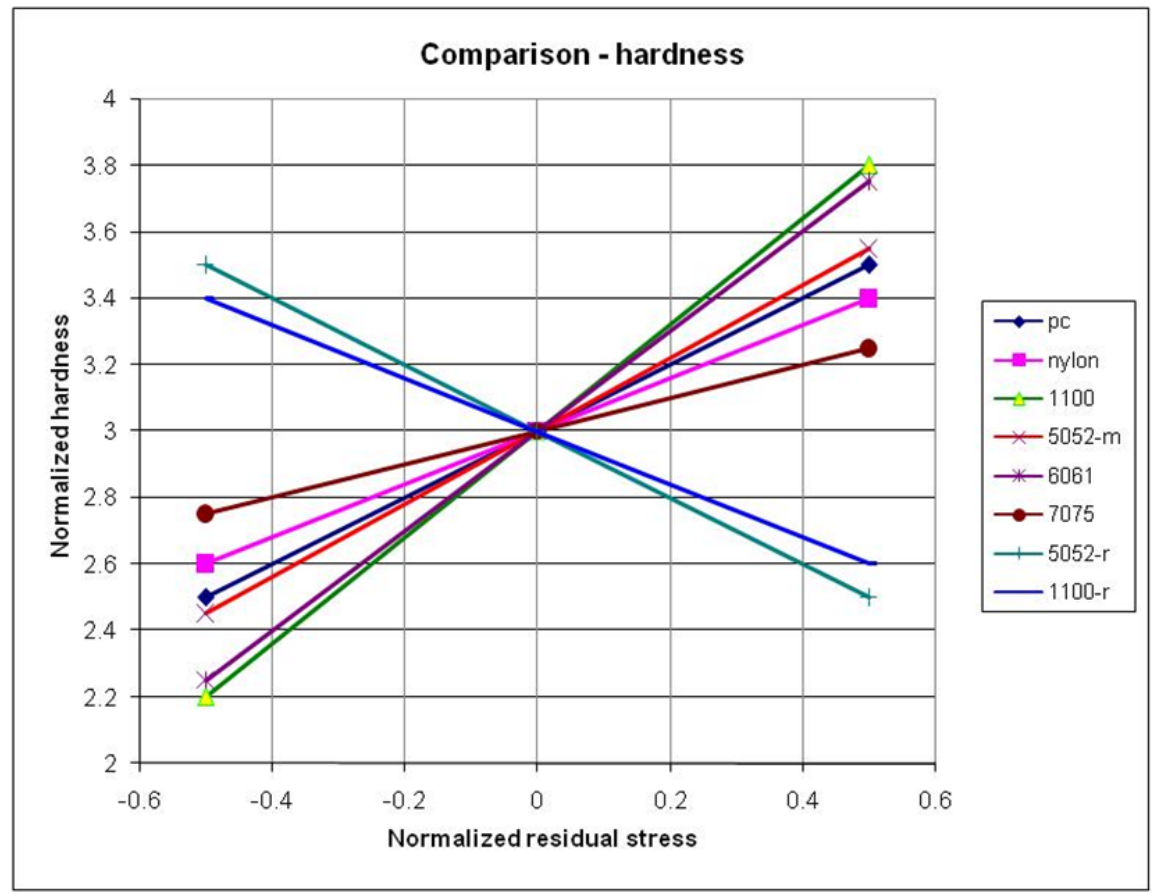

Fig 9: Normalized hardness vs Normalized residual stress

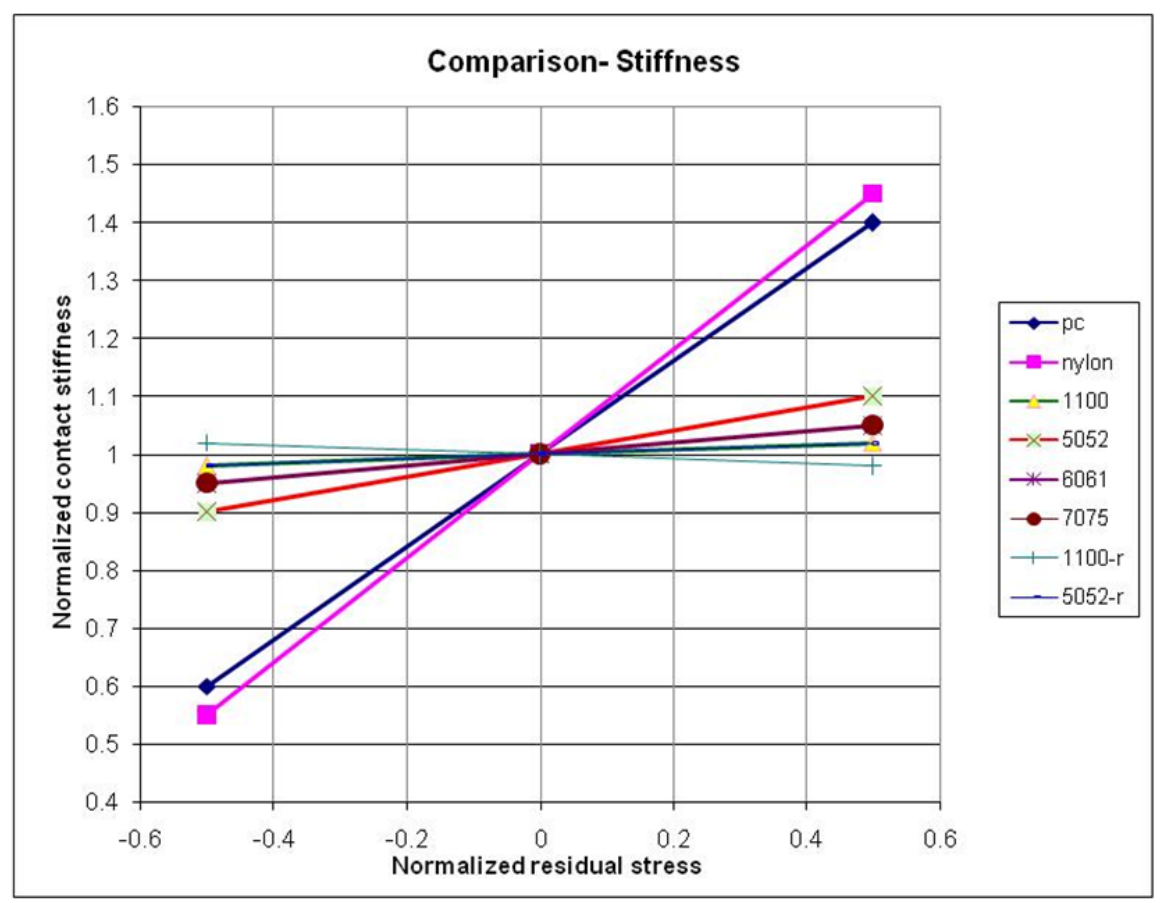

Fig 10: Normalized stiffness vs Normalized residual stress 


\section{CONCLUSIONS}

Micro-indentation experiment has been designed and developed to study the effect of varying in-plane biaxial residual stress on the mechanical properties of bulk materials. An attempt has been made to identify, quantify and remove the experimental errors in micro-indentation testing. The load frame compliance was estimated from the results of typical variable depth indentation tests on Aluminium specimen. The load over stiffness parameter was plotted against the indentation depth for the correct load frame compliance and was used to correct the modulus at higher indentation depths. From the test results obtained from indentation testing of different grades of Aluminium and plastics, it is observed that the in-plane biaxial tensile and compressive residual stresses have a visible effect on the mechanical properties (hardness and modulus) of the bulk materials measured using indentation tests. This can be used in the applications such as thermal barrier coatings where residual stresses are important due to material mismatch between coating and substrate. A simple micro-indentation test can tell exact information about the level of residual stresses in the thin coating surface.

\section{ACKNOWLEDGEMENT}

The part of this work was done in the University of Delaware as part of the research work done by author while he was there and hence author thanks Prof AM Karlsson for the kind help provided.

\section{REFERENCES}

1. Oliver W.C. and Pharr G.M., "An improved technique for determining hardness and elastic modulus using load and displacement sensing indentation experiments", Journal of Material Research, vol. 7(6), 1564-1583, 1992

2. Oliver W.C. and Pharr G.M., "Measurement of hardness and elastic modulus by instrumented indentation: Advances in understanding and refinements to methodology", Journal of Material Research, Vol. 19(1), pp.3-20, 2004

3. Pharr G. M and Bolshakov A., "Understanding nanoindentation unloading curves", Journal of Material Research, vol. 17(10), pp. 2660-2671, 2002

4. Bolshakov A. and Pharr G. M, "Influences of pile-up on the measurement of mechanical properties by load and depth sensing indentation techniques", Journal of Material Research, vol. 13(4), pp. 1049-1058, 1998

5. Hay J.L. and Pharr G.M., Instrumented indentation testing, ASM Handbook Vol. 8: Mechanical Testing and Evaluation, $10^{\text {th }}$ Edition, pp. 231-242, 2000

6. Beck U. and Griepentrog M., "Instrumented indentation test for determination of hardness and other plastic-elastic material properties, Reference Procedure", Federal Institute of Material Research \& Testing (BAM), 2005

7. Swadner J.G., Taljat B., Pharr G.M., "Measurement of residual stress by load and depth sensing indentation with spherical indenters", Journal of Material Research, Vol. 16(7),pp. 2091-2102, 2001

8. Carlsson S. and Larsson P. L., "On the determination of residual stress and strain filed by sharp indentation testing Part II: Experimental Investigation", Acta Materialia, vol. 49, pp. 2193-2203, 2001

9. Lee Y. H. and Kwon D., "Measurement of residual stress effect by nano-indentation on elastically strained (100) W", Scripta Materialia, vol. 49, pp. 459-465, 2003

10. Lee Y. H. and Kwon D., "Estimation of biaxial surface stress by instrumented indentation with sharp indenters", Acta Materialia, vol. 52, pp. $1555-1563,2004$

11. Tsui T. Y., Oliver W. C., and Pharr G. M., "Influences of stress on the measurement of mechanical properties using nanoindentation: Part I. Experimental studies in an aluminium alloy”, Journal of Material Research, vol. 11(3), pp. 752-759, 1996

12. Anton R.J., Miskioglu I. and Subhash G., "Determination of residual stress fields beneath a Vickers indentation using photoelasticlity", Experimental Mechanics, vol. 39(3), pp. 227-230, 1999

13. Bolshakov A., Oliver W. C., and Pharr G. M., "Influences of stress on the measurement of mechanical properties using nanoindentation: Part II. Finite element simulations", Journal of Material Research, vol. 11(3), pp.760-768, 1996

14. Carlsson S. and Larsson P. L., "On the determination of residual stress and strain filed by sharp indentation testing Part I: Theoretical and numerical analysis”, Acta Materialia, vol. 49, pp. 2179-2191, 2001

15. Suresh S. and Giannakopoulos A. E., "A new method for estimating residual stresses by instrumented sharp indentation", Acta Materialia, vol. 46(16), pp. 5755-5767, 1998

16. Chen Xi, Yan Jin and Karlsson A. M., "On the determination of residual stress and mechanical properties by indentation", Journal of Material Science \& Technology, vol. 416, pp. 139-149, 2006

17. P. Jadhav, A.M Karlsson, "Micro-Indentation experiments to measure in-plane residual stresses: Design methodology", $A I A A-A S M E$ annual technical symposium, Feb 2006, Pennsylvania

\section{Author Profile}

Prof. Prakash Jadhav did his PhD from The University of Mississippi, US and Post Doctorate from The University of Delaware, US. He has worked in industry for long time before joining as a Professor in his current job. He has $20+$ publications to his credit in international journals and conference proceedings 